\title{
A Proposed Dedicated Breast PET Lexicon: Standardization of Description and Reporting of Radiotracer Uptake in the Breast
}

\author{
Kanae K. Miyake ${ }^{1, *(D)}$, Masako Kataoka ${ }^{2}$, Takayoshi Ishimori ${ }^{2}$, Yoshiaki Matsumoto ${ }^{3,4}$, Masae Torii ${ }^{5}$, \\ Masahiro Takada ${ }^{3}$, Yoko Satoh ${ }^{6}$, Kazunori Kubota ${ }^{7}$ (D), Hiroko Satake ${ }^{8}$, Masahiro Yakami ${ }^{4}$, Hiroyoshi Isoda ${ }^{4}$, \\ Debra M. Ikeda ${ }^{9}$, Masakazu Toi ${ }^{3}$ and Yuji Nakamoto ${ }^{2}$
}

1 Department of Advanced Medical Imaging Research, Graduate School of Medicine Kyoto University, 54 Shogoin Kawahara-cho, Sakyo-ku, Kyoto-City 606-8507, Kyoto, Japan

2 Department of Diagnostic Imaging and Nuclear Medicine, Graduate School of Medicine Kyoto University, 54 Shogoin Kawahara-cho, Sakyo-ku, Kyoto-City 606-8507, Kyoto, Japan; makok@kuhp.kyoto-u.ac.jp (M.K.); ishimori@kuhp.kyoto-u.ac.jp (T.I.); ynakamo1@kuhp.kyoto-u.ac.jp (Y.N.)

3 Department of Breast Surgery, Graduate School of Medicine Kyoto University, 54 Shogoin Kawahara-cho, Sakyo-ku, Kyoto-City 606-8507, Kyoto, Japan; yoshiaki@kuhp.kyoto-u.ac.jp (Y.M.); masahiro@kuhp.kyoto-u.ac.jp (M.T.); toi@kuhp.kyoto-u.ac.jp (M.T.)

4 Preemptive Medicine and Lifestyle Related Disease Research Center, Kyoto University Hospital, 53 Shogoin Kawahara-cho, Sakyo-ku, Kyoto-City 606-8507, Kyoto, Japan; yakami@kuhp.kyoto-u.ac.jp (M.Y.); sayuki@kuhp.kyoto-u.ac.jp (H.I.)

5 Department of Breast Surgery, Japanese Red Cross Wakayama Medical Center, 4-20 Komatsubara-dori, Wakayama-City 640-8558, Wakayama, Japan; masaet@kuhp.kyoto-u.ac.jp

check for updates

Citation: Miyake, K.K.; Kataoka, M.; Ishimori, T.; Matsumoto, Y.; Torii, M.; Takada, M.; Satoh, Y.; Kubota, K.; Satake, H.; Yakami, M.; et al. A Proposed Dedicated Breast PET Lexicon: Standardization of Description and Reporting of Radiotracer Uptake in the Breast. Diagnostics 2021, 11, 1267. https:/ / doi.org/10.3390/diagnostics11071267

Academic Editor: Hidehiko Okazawa

Received: 23 May 2021

Accepted: 12 July 2021

Published: 15 July 2021

Publisher's Note: MDPI stays neutral with regard to jurisdictional claims in published maps and institutional affiliations.

Copyright: (c) 2021 by the authors. Licensee MDPI, Basel, Switzerland. This article is an open access article distributed under the terms and conditions of the Creative Commons Attribution (CC BY) license (https:// creativecommons.org/licenses/by/ $4.0 /)$.
6 Yamanashi PET Imaging Clinic, 3046-2 Shimokato, Chuo-City 409-3821, Yamanashi, Japan; ysatoh@ypic.jp

7 Department of Radiology, Dokkyo Medical University Saitama Medical Center, 2-1-50 Minamikoshigaya, Koshigaya-City 343-8555, Saitama, Japan; kubotard@dokkyomed.ac.jp

8 Department of Radiology, Nagoya University Graduate School of Medicine, 65 Tsurumai-cho, Showa-ku, Nagoya-City 466-8550, Aichi, Japan; shiroko@med.nagoya-u.ac.jp

9 Department of Radiology, Stanford University School of Medicine, Breast Imaging, 875 Blake Wilbur Drive, Stanford, CA 94305-5826, USA; dikeda@stanford.edu

* Correspondence: kanaek@kuhp.kyoto-u.ac.jp; Tel.: +81-75-751-3760; Fax: +81-75-771-9709

\begin{abstract}
Dedicated breast positron emission tomography (dbPET) is a new diagnostic imaging modality recently used in clinical practice for the detection of breast cancer and the assessment of tumor biology. dbPET has higher spatial resolution than that of conventional whole body PET systems, allowing recognition of detailed morphological attributes of radiotracer accumulation within the breast. ${ }^{18}$ F-fluorodeoxyglucose $\left({ }^{18} \mathrm{~F}-\mathrm{FDG}\right)$ accumulation in the breast may be due to benign or malignant entities, and recent studies suggest that morphology characterization of ${ }^{18}$ F-FDG uptake could aid in estimating the probability of malignancy. However, across the world, there are many descriptors of breast ${ }^{18}$ F-FDG uptake, limiting comparisons between studies. In this article, we propose a lexicon for breast radiotracer uptake to standardize description and reporting of image findings on dbPET, consisting of terms for image quality, radiotracer fibroglandular uptake, breast lesion uptake.
\end{abstract}

Keywords: breast cancer; positron emission tomography; dedicated breast PET; breast PET; highresolution PET; lexicon; ${ }^{18}$ F-fluorodeoxyglucose

\section{Introduction}

High-resolution positron emission tomography dedicated for the breast is a modern diagnostic imaging modality used in clinical practice for the detection of breast cancer and assessment of tumor biology, and is expected as a promising tool to support the evolution of molecular imaging [1]. High-resolution breast PET systems are PET systems that examine both breasts using detectors proximal to the breast, which were designed to have a higher spatial resolution and higher sensitivity for emitted radiation compared with conventional 
whole-body PET systems (WB-PET) [2]. High-resolution PET systems for the breast are generalized into two types: (1) "positron emission mammography (PEM)", a dual-head system compressing the breast mildly and providing limited-angle tomographic images [3], and (2) a fully tomographic type, representatively ring-type dedicated breast PET (dbPET), a newer generation of breast PET systems acquiring complete three-dimensional data and providing fully tomographic images in any direction $[4,5]$. While WB-PET has a limited value in evaluating, characterizing, and staging lesions within the breast due to its low spatial resolution, studies have shown that PEM or ring-type dbPET has a superior performance to WB-PET in the detection of breast cancers [6-10]. However, because of its high spatial resolution, dbPET detects and displays radiotracer accumulations that were previously unnoticeable, leading to the detection of a larger number of benign lesions and possibly leading to an increase in false-positive examinations.

Breast cancer is a heterogenous disease entity and has a wide variety of phenotypes. The imaging appearance of breast cancers is diverse with significant overlaps between benign and malignancy. This problem of imaging look-alikes was recognized on conventional X-ray, ultrasound, and dynamic contrast-enhanced magnetic resonance imaging (MRI) studies of the breast, with many publications identifying specific imaging features of breast cancers and useful findings to differentiate or characterize breast lesions, with several investigators generating methods to stratify the probability of malignancy according to the image-based interpretation. Because of the wide variety of naming methods and because of the need to standardize reporting, the American College of Radiology (ACR) organized a group of breast imaging experts to glean known scientific imaging publications for the best-known imaging features of breast lesions based on data. The result was the Breast Imaging-Reporting and Data System (BI-RADS) for mammography, ultrasound, and MRI of the breast. BI-RADS provides terminology, called a lexicon, to describe and report imaging findings in a standardized way, and to provide final assessment classifications linked with specific management recommendations [11]. BI-RADS serves as a common language that clearly and consistently conveys findings and assessments from radiologists to referring physicians and patients. Standardized breast background and lesion reporting supports data collection and allows comparison between studies around the world. We believe this concept may be applied to high-resolution PET dedicated for the breast, which allows identifying qualitative features of breast uptake because of its improved spatial resolution. Adding morphological assessment may help differentiate malignant uptake and benign uptake to reduce false positives caused by improved uptake detection in benign breast tissue and/or breast lesions. In 2011, Narayanan et al. first reported the value of feature analysis of breast uptake using PEM with ${ }^{18} \mathrm{~F}$-fluorodeoxyglucose $\left({ }^{18} \mathrm{~F}-\mathrm{FDG}\right)$, and demonstrated morphological uptake features of additional lesions in patients with breast cancer were significant predictors of malignancy [12]. In recent years, there have been several reports that suggest the usefulness of breast lesion morphological assessment using a ring-type dbPET with ${ }^{18}$ F-FDG [13-16]. However, to establish and facilitate a comprehensive approach of breast cancer diagnosis using dbPET, there is now a critical need to standardize reporting and descriptors of uptake features.

This paper proposes a dbPET lexicon to describe breast radiotracer uptake on dbPET, and to help in reporting dbPET findings. Many of the terms are similar to the ACR BI-RADS descriptors in order to harmonize with their terminology; however, some descriptors are unique to dbPET given PET's specific imaging parameters. Typical images of ring-type dbPET with ${ }^{18}$ F-FDG for each lexicon are provided.

\section{Methods}

2.1. dbPET Imaging Protocols and Display

2.1.1. Study Protocols and Parameters

Requirements before examinations should follow the general recommendations of PET studies, e.g., at least $4 \mathrm{~h}$ fasting for ${ }^{18}$ F-FDG $[17,18]$. 
The report should include the type of dbPET device, the imaging acquisition and reconstruction protocols, including the type and injection dose of radiotracer, blood sugar level at injection for ${ }^{18}$ F-FDG, waiting time, emission scan duration, and the reconstruction parameters. Any suboptimal conditions, such as poor positioning and body movement, should be noted in the report, since they potentially influence the degree and the appearance of uptake.

The report should include the study indication, pertinent demographic data, and patient clinical history (examples: gender, patient age, menstrual and menopausal status, medication/exogenous hormone or anti-hormone therapy, breast cancer history, breast intervention and breast reconstruction, and family history of breast cancers and ovarian cancers), as well as any prior examinations and study dates.

\subsection{2. dbPET Image Display}

Maximum intensity projection (MIP) images of dbPET should be initially assessed with a fixed window width; the recommended lower and upper thresholds are 0 and 3-4, respectively, in standard uptake value (SUV). With implementation of attenuation and scatter corrections, SUV is measurable with some fully tomographic dbPET systems [2,4,5]. It is recommended to display the paired mediolateral (ML) MIP views and the paired craniocaudal (CC) MIP views to compare right and left breasts and add an SUV scale on the side of the images. If the uptake of fibroglandular tissue or breast lesions is intense, it may be necessary to compose additional MIP images with a higher upper threshold to appreciate the dynamic range. Subsequently, tomographic images of each breast should be assessed, adjusting the window to the level to visualize internal properties of the breast or of lesions if needed.

\section{2. dbPET Lexicon, Version 1.0}

The lexicon includes descriptors for image quality, background fibroglandular uptake (bFGU), and breast lesions (Table A1 in Appendix A). Image quality and bFGU are routinely assessed regardless of the presence or absence of breast lesions.

\subsubsection{Image Quality}

Assessment of image quality is the first step to describe dbPET findings. Noise and field of views (FOV) are included in this assessment.

Noise tends to be relatively higher and the signal-to-noise ratio tends to be lower at the edge of the detector where the gamma-ray coincidences from the annihilation of positrons decrease. Noise level is categorized using the 4-point scale; minimal, mild or limited to the edge of FOV, moderate, and marked (Figure 1). Minimal noise means absence of noise or almost no noise. Mild or limited to the edge of FOV noise includes mild noise, and noise that is relatively strong but localized to the near edge of FOV and does not affect most of the breast. Moderate noise is moderately significant noise that extends over the breast parenchyma, not limited to the edge of FOV. Marked noise is significant noise that has high potential to mask lesion uptake.

FOV is determined by how much of the breast is included in the FOV of dbPET. The retromammary space is used as a marker to estimate the FOV visualization of breast tissue because the pectoral muscles are usually not included within the FOV of dbPET, which has a wider blind area than mammography. The FOV of dbPET is categorized as full, almost full, partial, and limited (Figure 2). Inclusion of the retromammary space is used to categorize a full, almost full, or partial FOV, describing when the retromammary space is visualized entirely (full), mostly (almost full), or partially to poorly (partial). A limited FOV means that the breast within the FOV is extremely small due to small breast size, poor positioning, or partial mastectomy, etc. It should be noted that there is a possibility that a part of the breast is still excluded from the FOV even in the case of a full FOV. 

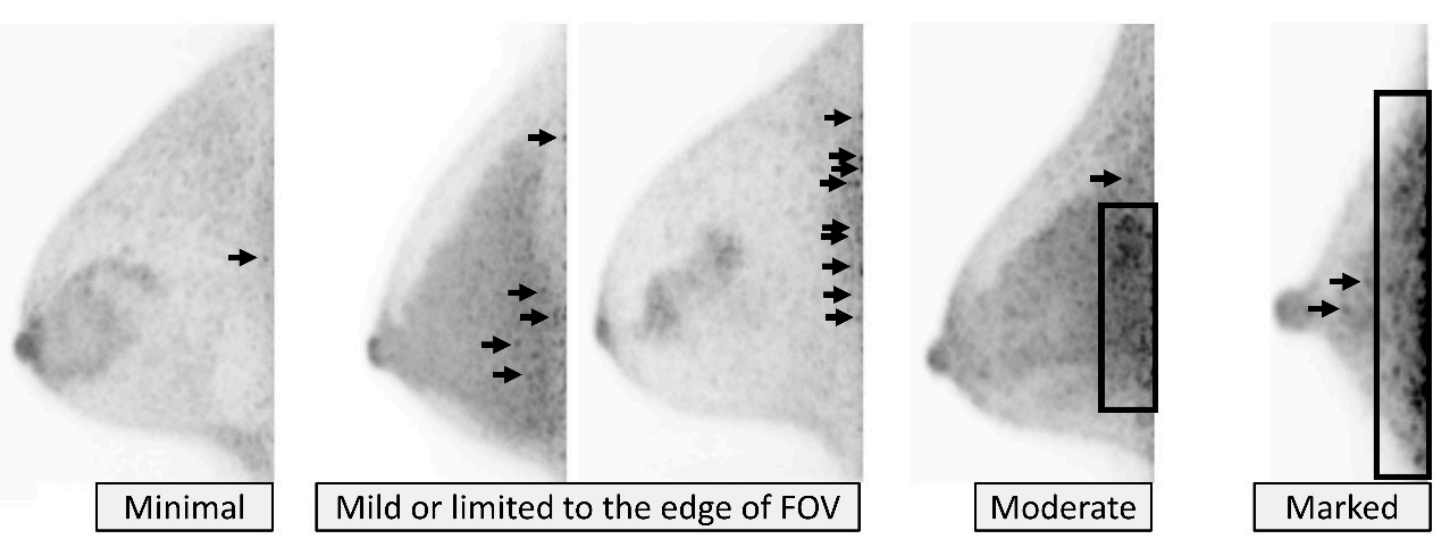

Figure 1. Image quality: Noise. From left to right, minimal, mild (categorized as "mild or limited to the edge of field of view $\left.(F O V)^{\prime \prime}\right)$, limited to the edge of FOV (categorized as "mild or limited to the edge of FOV"), moderate, and marked on MIP images. Arrows and rectangles indicate noise.
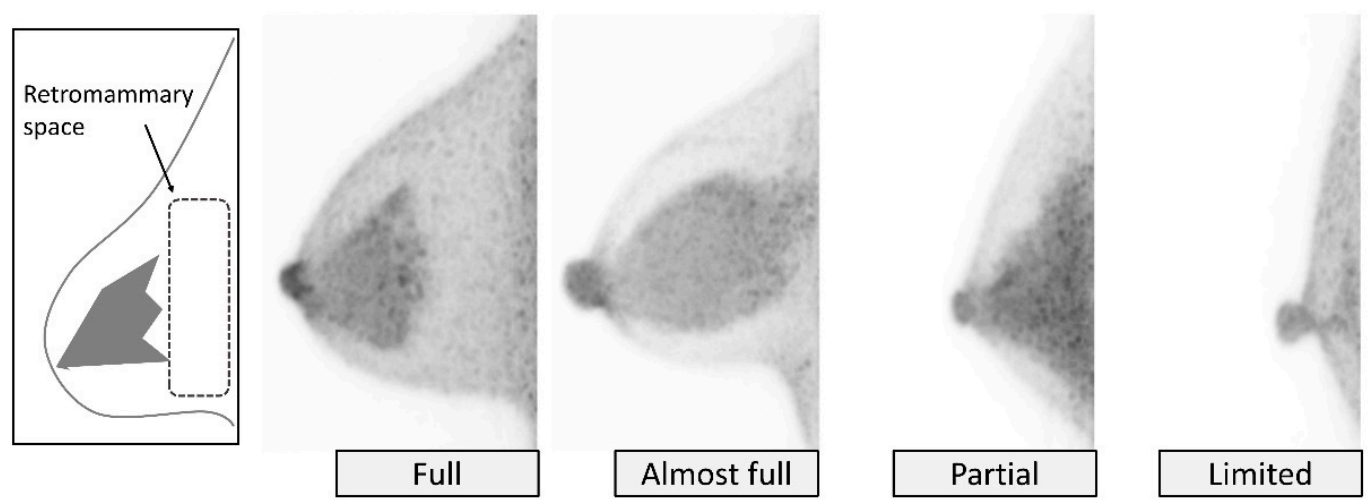

Figure 2. Image quality: Field of view (FOV). FOV is classified using retromammary space (see schematics) as hallmark. From left to right, full, almost full, partial, and limited on MIP images.

If there are special notes regarding imaging conditions that can degrade image quality, such as poor positioning, body movement, and change of protocols, we recommend that these be described within the study comments.

\subsubsection{Background Fibroglandular Uptake (bFGU)}

Fibroglandular tissue usually has higher ${ }^{18} \mathrm{~F}-\mathrm{FDG}$ accumulation compared with fat, thus is seen as FDG-avid structure on dbPET. Background breast fibroglandular uptake (bFGU) is assessed in terms of fibroglandular amount, uptake intensity, distribution, and symmetry. It is important to report bFGU because intense bFGU may hide breast cancers.

bFGU extent, as a report of the amount of radiotracer-avid background breast tissue in the breast by volume, is categorized with the 5-point scale as none, small, relatively small, relatively large, and large, corresponding to $0 \%$ (none), $>0 \%$ to $\leq 25 \%$ (small), $>25 \%$ to $\leq 50 \%$ (relatively small), $>50 \%$ to $\leq 75 \%$ (relatively large), and $>75 \%$ to $\leq 100 \%$ (large), respectively, of the assumed fibroglandular tissue occupying the glandular areas of the breast (Figure 3). No bFGU (none) may be rare but can be occasionally seen, e.g., in aged breasts entirely replaced with fat, or in women with mastectomies. When the category none is chosen, evaluation of intensity and homogeneity of bFGU will be omitted, and the reason why the breast tissue is absent should be reported. 


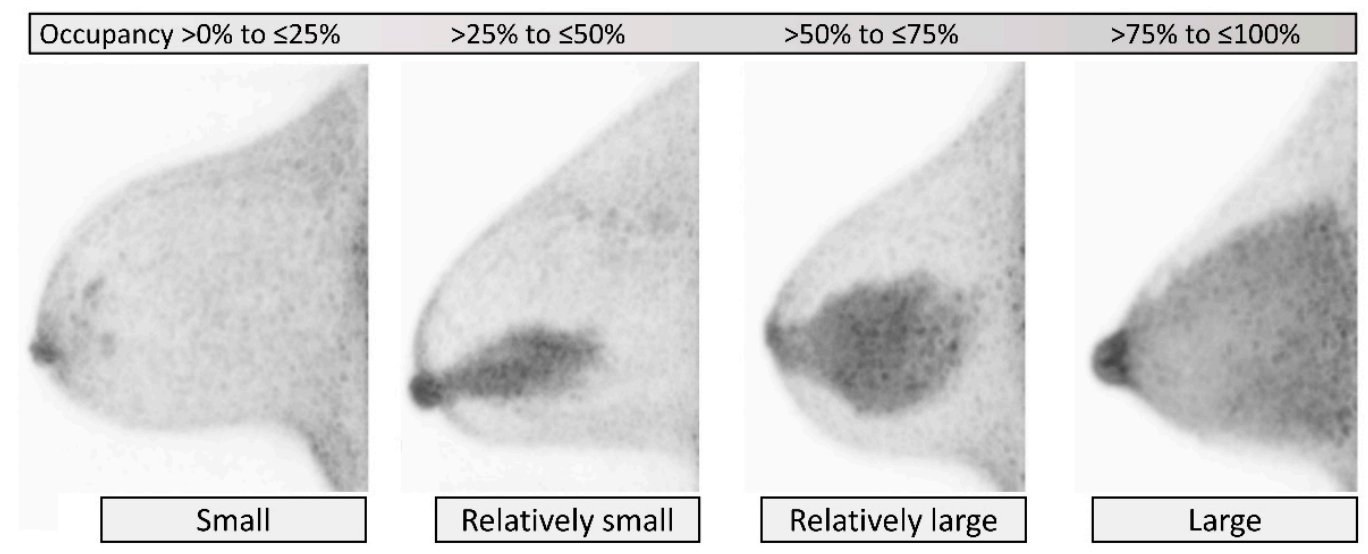

Figure 3. Background fibroglandular uptake (bFGU): Extent. From left to right, small, relatively small, relatively large, and large on MIP images. No uptake (none), another category, is not shown. Amount is classified based on the approximate occupancy of bFGU in the assumed fibroglandular tissue in the breast by volume.

bFGU intensity is categorized as faint, mild, moderate, and intense, corresponding to approximately $<1, \geq 1$ and $<2, \geq 2$ and $<3$, and $\geq 3$ of SUV as a guide, respectively (Figure 4). In heterogeneous bFGU, we suggest that the bFGU be evaluated and reported at the approximate level of areas with the most intense accumulation in the breast. In most cases, bFGU intensity may be classified as mild, or less commonly as moderate or faint. Intense bFGU may be seen in lactating breasts or pathological breasts. Intense bFGU can mask lesion uptake. If there is significant noise on the image, intensity of bFGU should be assessed in areas that are not affected by noise.

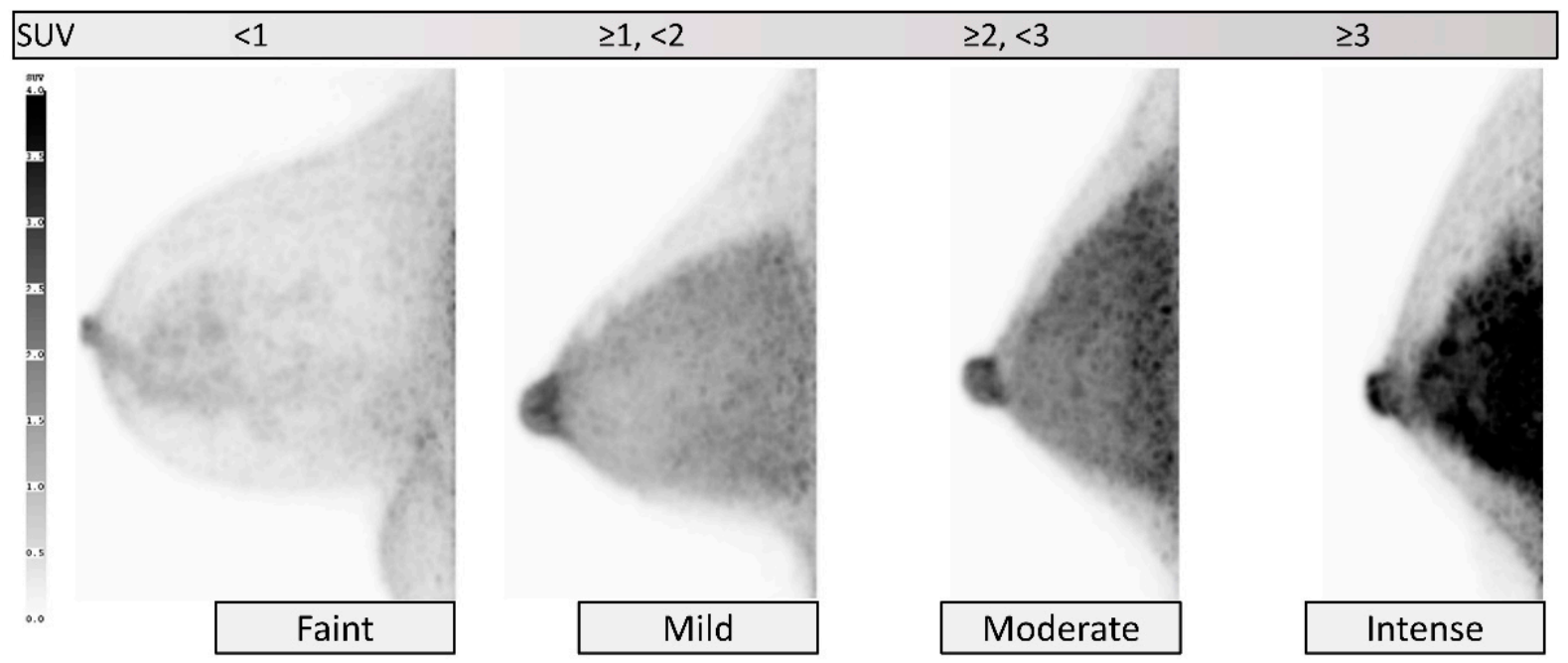

Figure 4. Background fibroglandular uptake (bFGU): Intensity. From left to right, faint, mild, moderate, and intense on MIP images. Intensity is classified using SUV scale as a guide.

Distribution of bFGU in the breast is categorized as homogeneous and heterogeneous (Figure 5). Homogeneous means almost uniform and confluent uptake of the radiotracer-avid fibroglandular tissue. Heterogeneous primarily means uneven distribution of radiotraceravid fibroglandular tissue due to the mixture of fat (i.e., heterogeneous, scattered, islandlike, or patchy distribution), but sometimes could be due to uneven uptake level of the fibroglandular tissue itself. 

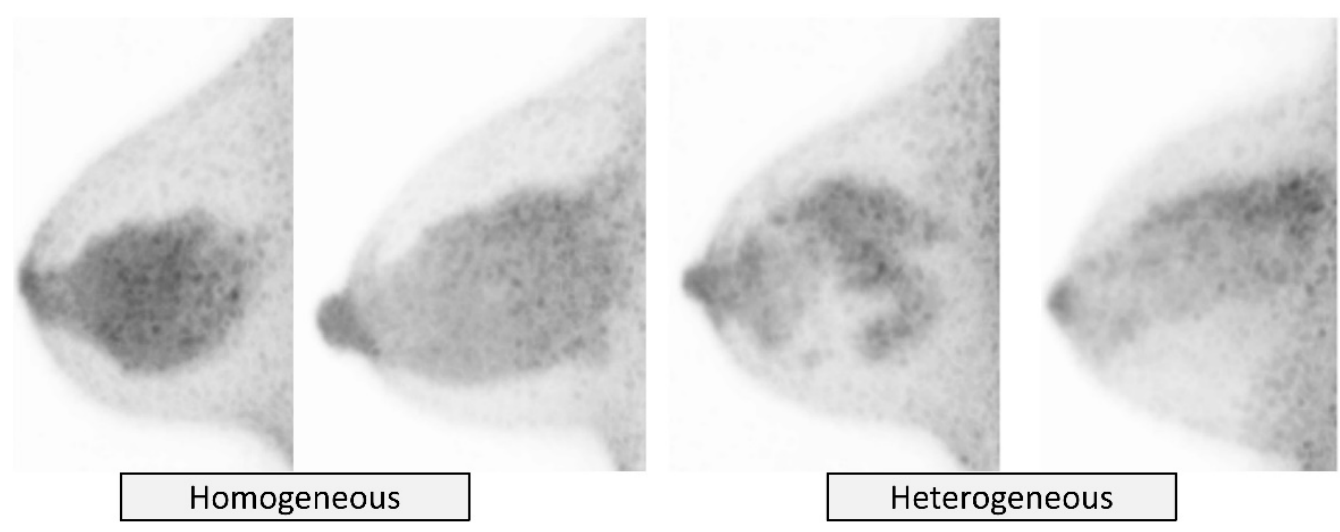

Figure 5. Background fibroglandular uptake (bFGU): Distribution. Homogeneous (left two) and heterogeneous (right two) on MIP images.

Symmetry of bFGU in comparison of the left and right breasts is categorized as symmetric or asymmetric (Figure 6). The routine evaluation of distribution and symmetry may be helpful to allow earlier recognition of a lesion that exhibits unremarkable uptake, which is often difficult to distinguish from bFGU, such as ductal carcinoma in situ (DCIS).
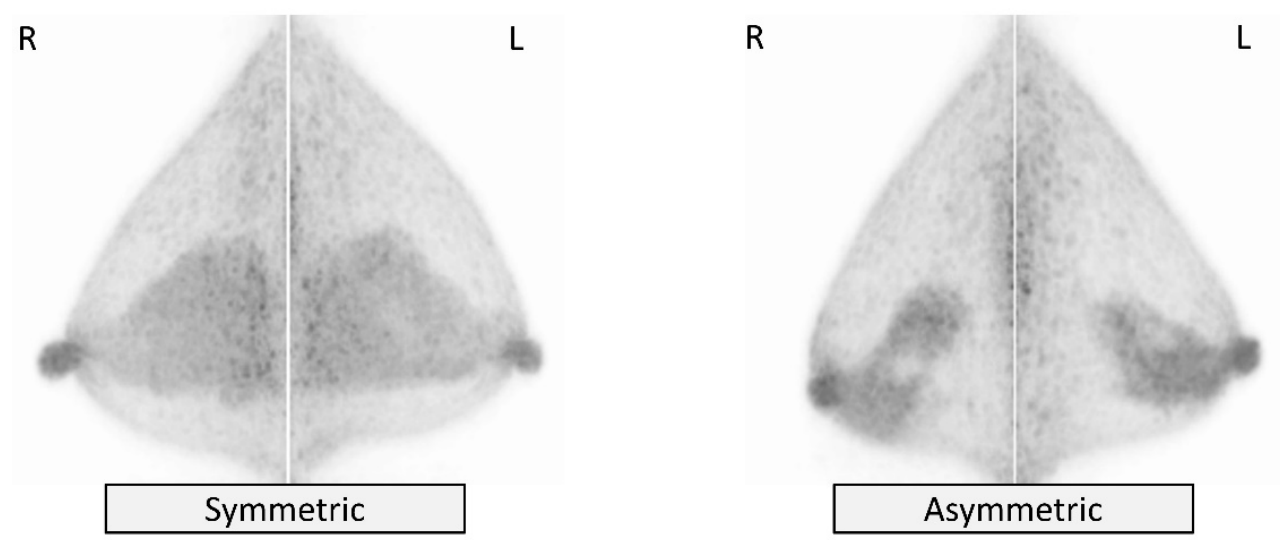

Figure 6. Background fibroglandular uptake (bFGU): Symmetry. Symmetric (left) and asymmetric (right) on MIP images.

If there are special notes such as post-mastectomy, post-reconstruction, we recommend that these be described in the indication and in the comments.

\subsubsection{Breast Lesion}

A lesion is defined as an area of abnormal uptake that is unique and different from the bFGU.

Lesions are classified as a focus, mass uptake (MU), and non-mass uptake (NMU) based on the three-dimensional morphologic features.

A focus is a dot-like small uptake that is difficult to characterize further. Although it is difficult to measure the exact size on PET and size threshold is not strictly defined as a criterion, a focus is roughly smaller than $5 \mathrm{~mm}$ as a guide in a fixed window of 0 to 3-4 on ${ }^{18}$ F-FDG dbPET.

Mass uptake (MU) is uptake larger than $5 \mathrm{~mm}$ composed of a three-dimensional uptake finding, which usually has relatively abrupt margins.

Non-mass uptake (NMU) is uptake that has a pattern different from that of the bFGU and has a shape that cannot be called a focus or a mass. NMU includes uptake with its boundary intensity shifting gradually toward the intensity of the background or is composed of tightly gathered multiple foci in one area.

For reporting of a breast lesion, we propose describing the lesion location and uptake intensity using the following descriptors. 
Descriptions of the lesion location include breast laterality (right or left breast), breast quadrant (upper outer quadrant towards the axilla (UOQ), upper inner quadrant (UIQ), lower outer quadrant $(L O Q)$, lower inner quadrant $(L I Q)$, and around or posterior to the nipple (retroareolar)), clock-face location, lesion depth (anterior, middle, and posterior third of the breast), and the distance of the lesion from the nipple (Figure 7).
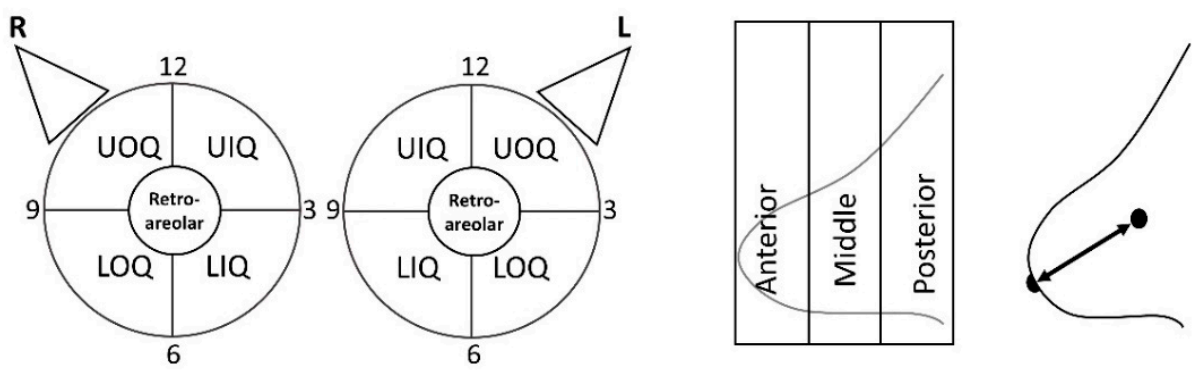

Figure 7. Lesion: Location. Schematics of quadrant and clock-face location (left), depth (middle), and the distance of the lesion from the nipple (right). $U I Q=$ upper inner quadrant, $L I Q=$ lower inner quadrant, $U O Q=$ upper outer quadrant, $L O Q=$ lower outer quadrant.

Specifically, the lesion clock-face location is described as if the patient is facing the interpreter and each breast is on the face of a clock. In both the right and left breasts, lesions at the cranial aspect of the breast at the level of the nipple would be described as at the 12 o'clock position, while lesions at the caudal aspect of the breast at the level of the nipple would be described as at the 6 o'clock position of the breast. However, lesions in the right breast in the upper outer quadrant towards the axilla (UOQ) would be at the 10 o'clock position, whereas lesions in the UOQ of the left breast would at the 2 o'clock position.

Qualitative uptake intensity level is determined based on the visual assessment of the intense parts of the lesion using SUV scale as a guide, and categorized as no abnormal uptake or none, mild (approximately SUV <2), moderate (approximately SUV $\geq 2$ and $<3$ ), and intense (approximately SUV $\geq 3$ ) (Figure 8). Quantitative assessment, using SUV, can be applied but is not mandatory. It should be noted that SUVmax is a pixel value, which can overestimate uptake degree and be enhanced by the effect of noise, especially on high-resolution dbPET images. None or no abnormal intensity in the location of a known breast lesion may be difficult to be identified using dbPET alone, and should be reserved to assess a specific lesion in comparison with other imaging modalities. As an example, if there are breast calcifications highly suspicious for malignancy on a mammogram and the dbPET shows no abnormal uptake in the same location, one may report the intensity of the dbPET in the location of the known suspicious finding as having "no abnormal uptake" or that the uptake intensity of the dbPET is "none" in the location of the known suspicious finding.

As described earlier, all lesions should be initially assessed on MIP using a fixed window (recommendation: 0 to $3-4$ for ${ }^{18}$ F-FDG), and then further evaluated with tomographic images using an adjusted dynamic range to see detailed ${ }^{18}$ F-FDG uptake pattern within tumors. Particularly, for malignant lesions with intense uptake, it is important to adjust the dynamic range to assess the lesion morphology and internal uptake details.

\section{Focus}

Focus is a dot-like area of uptake that is difficult to characterize further, and is usually smaller than $5 \mathrm{~mm}$ in a fixed window of 0 to $3-4$ for ${ }^{18}$ F-FDG. A focus can include noise, unless noise is reliably differentiated from true abnormal uptake.

The focus descriptor is subdivided into a single focus or multiple foci (Figure 9). A single focus is one, solitary dot-like area of uptake. Two foci are described separately as a different single focus for each. The term "multiple foci" describes three or more foci scattered in the breast independently from the distribution of glandular tissue lobes. If three or more 
foci gather closely together or appear along a glandular lobe, they are described as NMU instead of multiple foci.
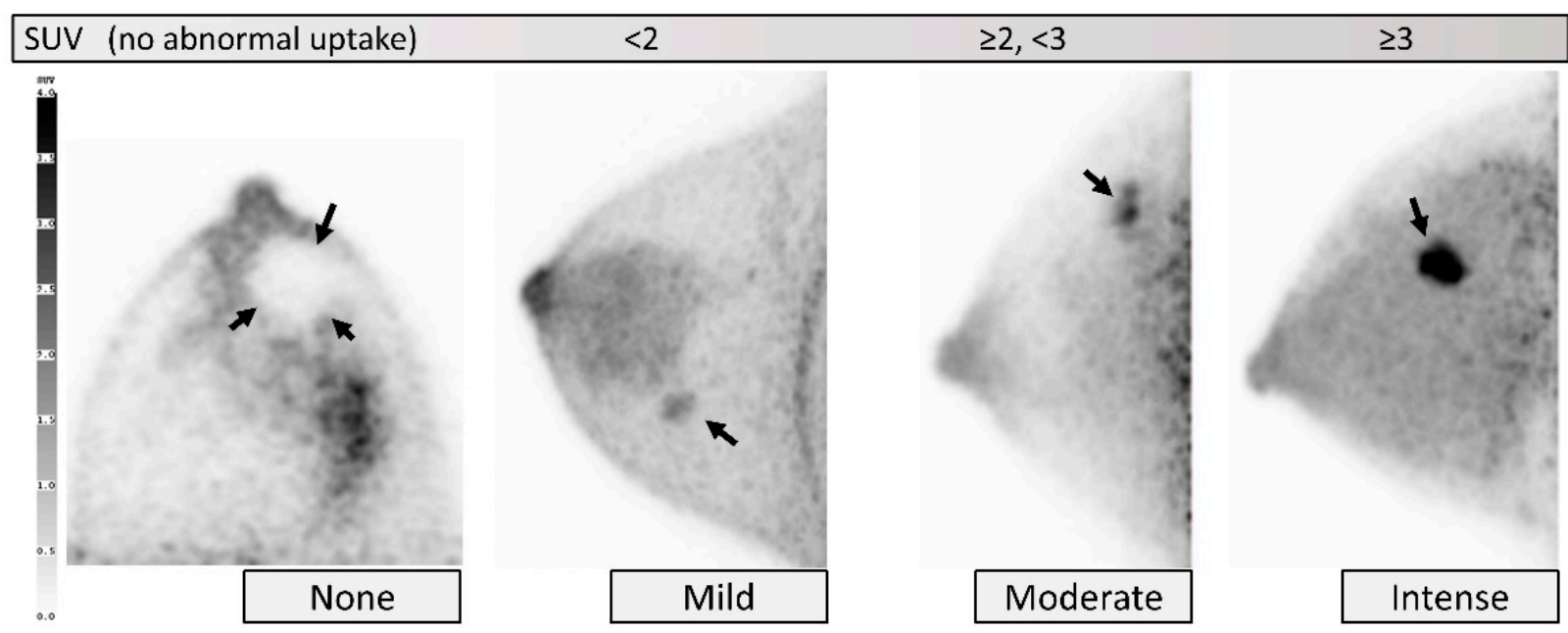

Figure 8. Lesion: Intensity. From left to right, none (cyst; tomographic image), mild (category 2 on ultrasound; MIP), moderate (intraductal papilloma; MIP), and intense (invasive ductal carcinoma; MIP). Intensity is classified using SUV scale as a guide. Arrows indicate target lesions.

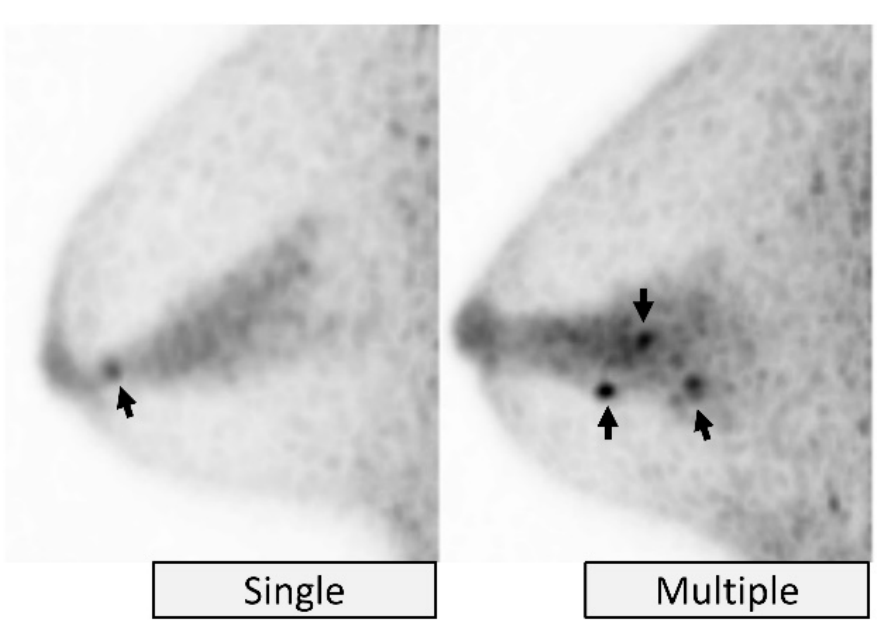

Figure 9. Focus. Single (left, intraductal papilloma), and multiple (right, usual ductal hyperplasia) on MIP images. Arrows indicate foci.

\section{Mass Uptake (MU)}

Mass uptake $(M U)$ describes a three-dimensional mass-like area of uptake that usually has distinct edges compared to the bFGU, and is described by its shape and internal pattern.

A mass shape is categorized as oval, round, lobulated, or irregular, based on the features of both the overall mass uptake shape and margins (Figure 10). Oval or round mass uptake has smooth margins, and may be accompanied with up to two mild undulations with a dull angle. Lobulated mass uptake also has smooth margins but has apparent lobulations with clear sharp angles with three or more undulations. Irregular masses have irregular margins (sawtooth, spiculated, angular, or indistinct) or are those that have irregular extensions into the surrounding tissue. 


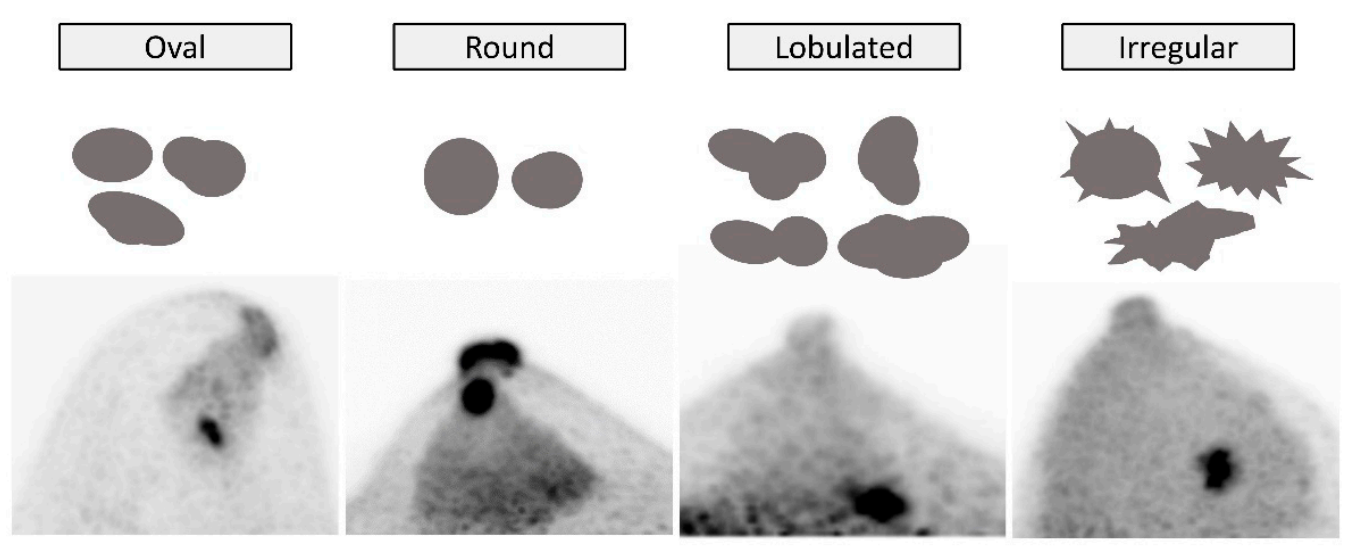

Figure 10. Mass uptake: Shape. From left to right, oval (no malignancy on biopsy), round (no histological diagnosis), lobulated (no histological diagnosis), and irregular (invasive ductal carcinoma) on MIP images with schematics.

The mass internal pattern is categorized as homogeneous, heterogeneous, or rim uptake (Figure 11). Homogeneous uptake describes uptake that is of uniform intensity throughout the lesion. Heterogeneous uptake describes variable uptake intensity within the lesion. Rim uptake describes unique heterogeneous moderate or intense uptake along the edges of the lesion or along the lesion "rim" with the central area of the lesion showing no or mild abnormal uptake. Rim uptake includes either a full rim (uptake highest along all lesion edges) or a partial rim (higher uptake along only part of the lesion edge). Heterogeneous uptake within and up to the lesion edge other than rim uptake is categorized as heterogeneous.

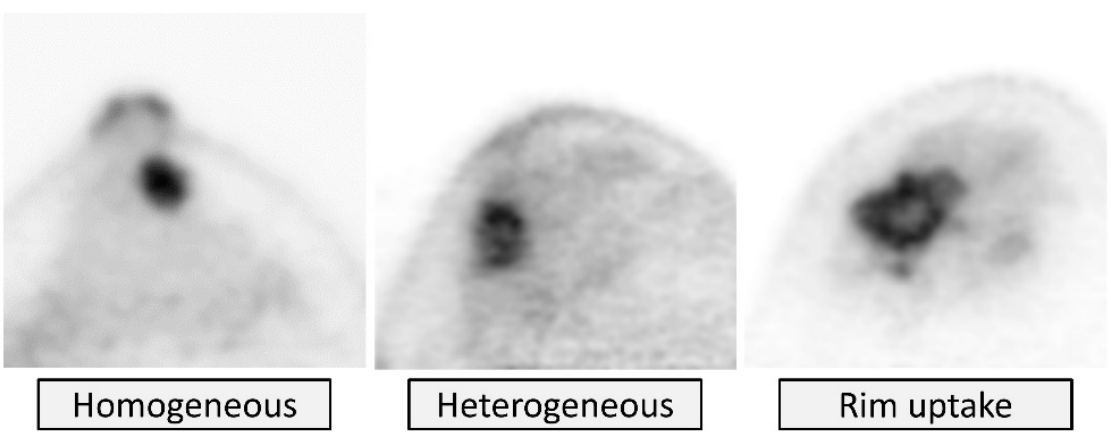

Figure 11. Mass uptake: Internal pattern. From left to right, homogeneous (no histological diagnosis), heterogeneous (invasive ductal carcinoma), and rim uptake (invasive ductal carcinoma) on tomographic images.

\section{Non-Mass Uptake (NMU)}

Non-mass uptake (NMU) is defined as abnormal uptake unique and different from that of the background fibroglandular glandular tissue (bFGU), larger than $5 \mathrm{~mm}$ and with a shape that cannot be called a mass or a focus. NMU includes uptake with an indistinct boundary and an accumulation intensity shifting gradually lower toward the bFGU (exhibiting one or more uptake grades lower than that of central part). NMU also describes multiple foci that are closely gathered together. NMU descriptors include both the NMU distribution and internal accumulation pattern.

NMU distribution descriptors include focal, linear, segmental, regional, multiple regions, and diffuse (Figure 12). Linear or segmental distribution may indicate extension of lesions along breast glandular lobes and ducts. Focal or regional distribution means localized NMU; focal $=$ less than $25 \%$ of quadrant, regional $=$ equal or more than $25 \%$ of quadrant. Multiple regional NMU distribution is defined as NMU that scatters in three or more locations, independently from the distribution of glandular lobes. When using this description for uptake consisting of mixtures of foci, the uptake pattern should include at least one location of NMU. Diffuse NMU describes NMU that almost continuously and widely extends over 
the whole breast. Examples of focus, multiple foci, NMU, multiple regional NMU, and diffuse NMU are illustrated in Figure 13.

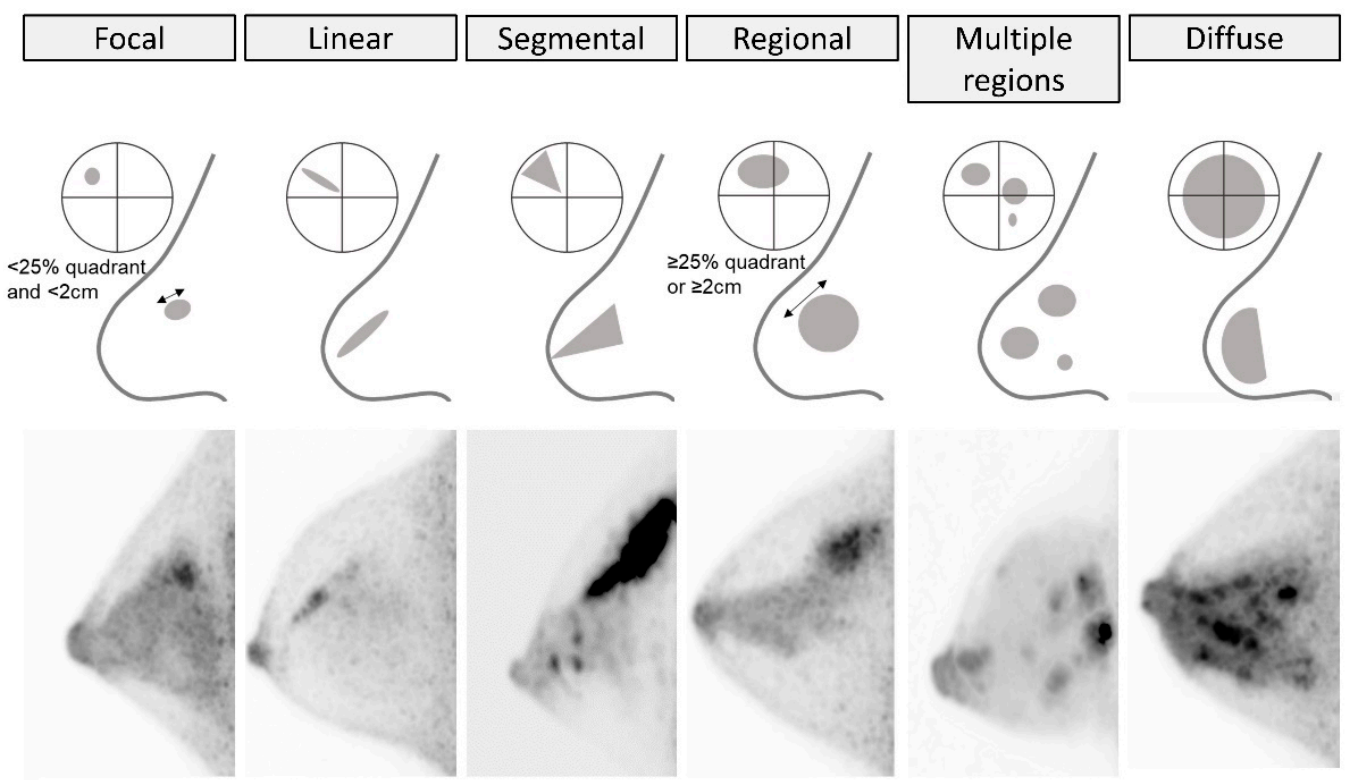

Figure 12. Non-mass uptake (NMU): Distribution. From left to right, focal (fibrocystic disease), linear (invasive ductal carcinoma), segmental (ductal carcinoma in situ and invasive ductal carcinoma), regional (radial sclerosing lesion), multiple regions (invasive ductal carcinoma), and diffuse (lymphocytic lobulitis) on MIP images with schematics. The double-headed arrows indicate the diameter of a lesion.

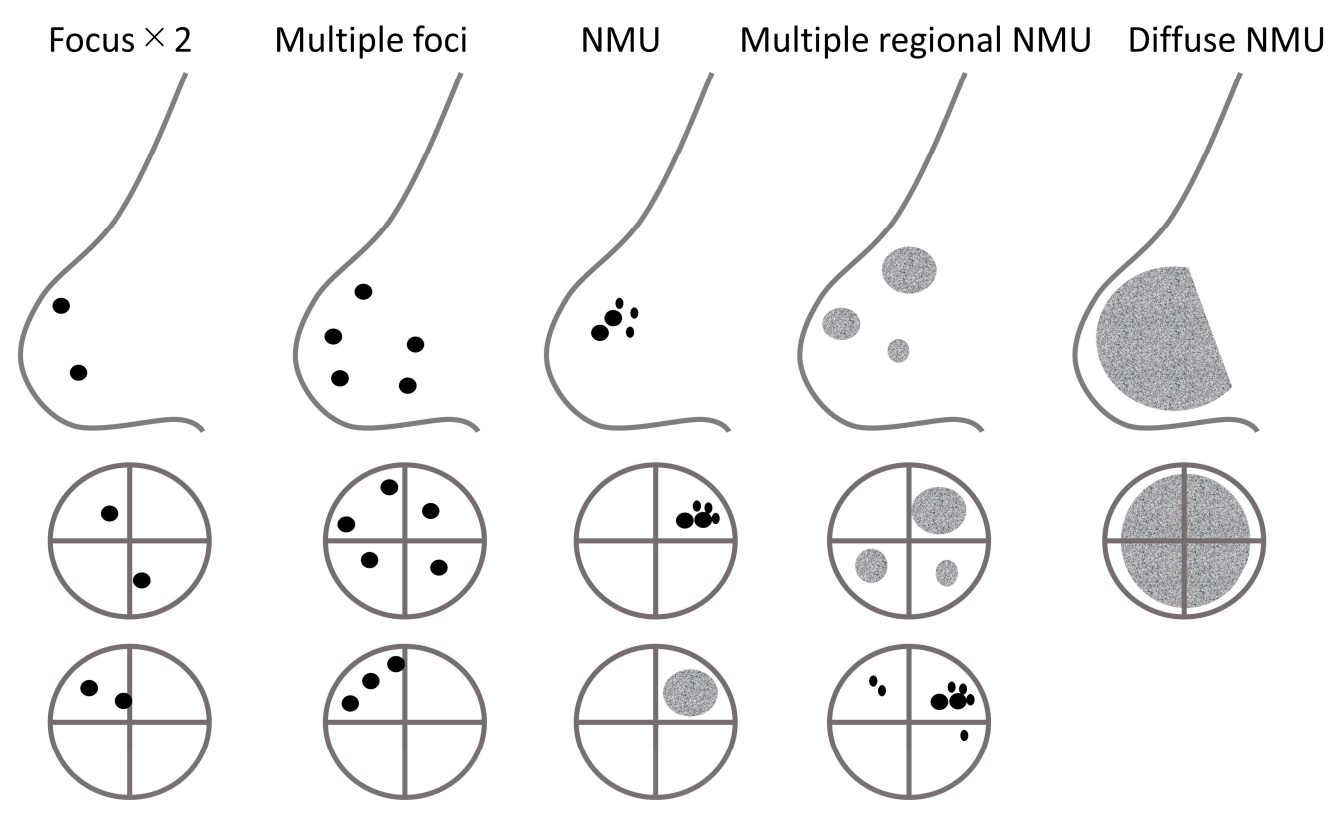

Figure 13. Schematics of focus, multiple foci, NMU, multiple regional NMU, and diffuse NMU.

The internal pattern of NMU is categorized as homogeneous, characterized by uniform uptake, or heterogeneous, characterized as non-uniform uptake (Figure 14).

Associated Findings

Features associated with lesion uptake, when visualized, include nipple retraction, skin retraction, nipple uptake, skin uptake, and pectoral muscle/chest wall uptake. 


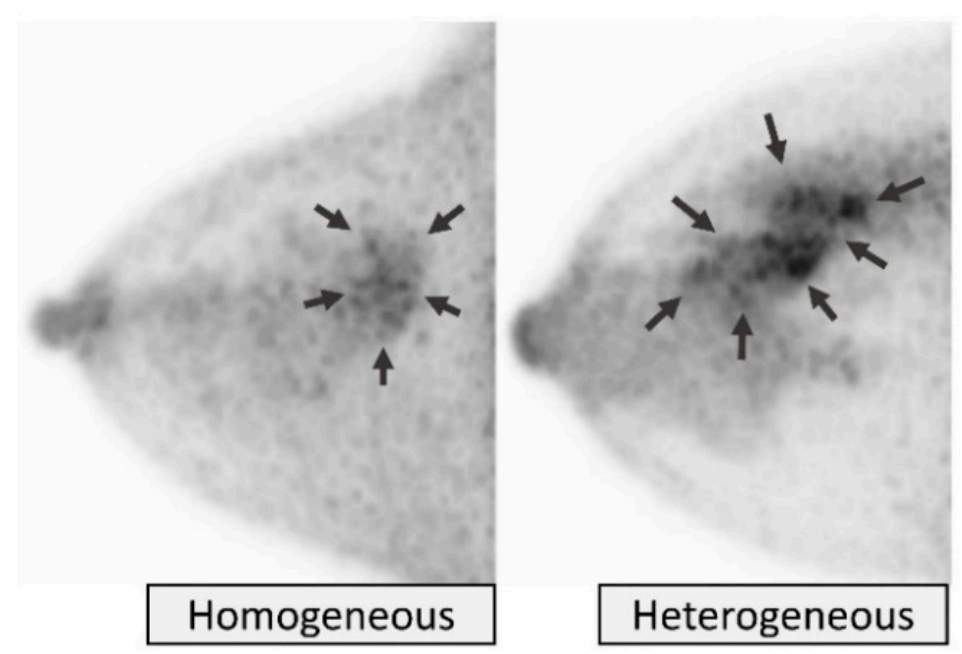

Figure 14. Non-mass uptake (NMU): Internal pattern. Homogeneous (left, ductal carcinoma in situ) and heterogeneous (right, no malignancy on biopsy). Arrows indicate NMU.

Special Findings

Cyst-like uptake describes a clear circular or ball-like uptake defect with or without surrounding uptake. This category is selected when a simple cyst, complicated cyst, or fat necrosis is strongly suspected or known by other imaging methods. If the uptake defect is not sharply marginated and difficult to define, or has rim uptake in a location without a known cyst, the uptake should be categorized as a mass with rim uptake.

A breast implant describes accumulation defects due to known breast implants. If there is elevated uptake around the implant, the increased uptake should be considered abnormal and described in the section of breast lesions.

\section{Discussion}

The clinical usefulness of morphological features of breast uptake on ring-type dbPET has been demonstrated by several reports. Satoh et al. found that mass uptake was significantly associated with malignancy compared to focus in 40 unexpected uptake lesions in 35 patients [13]. Sasada et al. showed that mass and focal/segmental non-mass lesions were significantly associated with breast cancer among 910 abnormal findings in 709 patients with breast cancer [14]. Masumoto et al. reported that heterogeneity in tumor uptake was associated with higher nuclear grade and higher Ki67 in breast cancers [15]. Sakaguchi et al. showed that rim uptake was associated with higher Ki67 and triplenegative tumor status [16]. Although the definitions of uptake features in these studies were not uniform, these reports suggest the addition of morphological feature analysis may be helpful in characterizing breast uptake, and could be a clue to resolve issues of false-positive reports caused by uptake in benign breast conditions. By collecting data using this lexicon with unified reporting criteria, we believe that features of breast cancer uptake may be clarified and the evidence of predictive values of each uptake feature may be established.

The ACR BI-RADS Atlas recommends that the interpretation of imaging findings use a final assessment category consisting of categories 0 to 6 , which represents the probability of malignancy and is linked to recommendations of management [11]. We encourage the adoption of this same system in the future, once the reproducibility and the usefulness of this lexicon has been validated and established.

It is an urgent task to identify the probabilities of malignancy of dbPET findings and establish the interpretational criteria of dbPET in breast cancer screening, which may be possible with the use of the standardized lexicon. Mammography and ultrasonography are conventional imaging modalities with established roles in the early diagnosis of breast cancer; however, some diagnostic limitations are known, such as mammography being less sensitive in dense breasts and ultrasonography being operator-dependent. MRI is 
known as the most sensitive modality for breast cancer, but has frequently resulted in false-positive diagnoses. Previously Berg et al. demonstrated that PEM with ${ }^{18}$ F-FDG was much more sensitive than conventional imaging (mammography and often targeted ultrasonography) ( $41 \%$ vs. $21 \%$ at lesion level) in the detection of additional cancers in the ipsilateral breast in 388 women with newly diagnosed breast cancer [19]. They also showed that PEM had improved specificity (79.9\% vs. $65.6 \%)$ compared with MRI, and was less likely to prompt unnecessary biopsies. A combination of high-resolution PET systems with standard breast imaging modalities may improve the breast cancer detection. In addition, with the development and application of various PET tracers, high-resolution PET systems will further extend the roles of imaging in breast cancer diagnosis.

In our dbPET lexicon, uptake intensity levels are graded using SUV scale as a guide. This grading system is designed to distinguish between relatively low levels of uptake, in order to differentiate the uptake levels of the normal fibroglandular tissue, that is, bFGU, as well as to identify and stratify breast lesions with mild or moderate uptake, especially those that need to be differentiated from small or low-FDG-avid breast cancers, on ${ }^{18} \mathrm{~F}$-FDG dbPET. According to a study by Nishimatsu et al., SUVmax of the contralateral breasts considered as normal in 140 patients with breast cancer ranged from 0.7 to 3.7 with a mean value of 1.8 on ${ }^{18} \mathrm{~F}$-FDG dbPET [20], supporting that our grading system for bFGU intensity is set to reasonable SUV levels. They also reported that SUVmax of 179 breast cancers widely varied with a range of 0.9 to 67.3. Sueoka et al. showed that the luminal A-like subtype, smaller size, and lower grade were significantly associated with lower SUVmax on ${ }^{18} \mathrm{~F}$-FDG dbPET [10]. Our lexicon is expected to contribute to picking up lesions with subtle contrast, including small or low-FDG-avid breast cancers, and further differentiating of these lesions.

The dbPET lexicon was created primarily assuming the use of ring-type dbPET with ${ }^{18}$ F-FDG. Previously, a lexicon for PEM with ${ }^{18}$ F-FDG [12] and for ${ }^{99 m}$ Tc-sestamibi dualhead gamma breast imaging [21] was proposed. Since ring-type dbPET has an advantage over PEM in that it provides complete three-dimensional images and tomographic images in any direction while quantifying SUVs, our descriptors include specific and detailed terms representing high-resolution three-dimensional breast lesion morphology and accumulation intensity using SUV as a guide. The concept and the information obtained by high-resolution PET systems, however, have much in common, and most parts of our lexicon may be potentially applicable to the other high-resolution PET devices, including PEM, as well as to various tracers. More research validating this reporting system's reproducibility and applicability is needed.

\section{Conclusions}

In this article, we propose a reporting lexicon of dbPET (version 1.0.) to standardize the reporting of dbPET and dbPET-detected breast findings, with an emphasis on descriptors of morphological features of normal breast fibroglandular tissue and breast lesions. With the advent of PET technology advances, the comprehensive assessment of breast uptake is possible based on three-dimensional morphology feature and uptake assessments.

Author Contributions: Conceptualization, K.K.M., M.K. and T.I.; resources, Y.M., M.T. (Masae Torii), M.T. (Masahiro Takada) and H.I.; data curation, M.Y.; writing-original draft preparation, K.K.M., M.K., T.I. and Y.N.; writing-review and editing, Y.S., K.K., H.S. and D.M.I.; supervision, M.T. (Masakazu Toi) and Y.N. All authors have read and agreed to the published version of the manuscript.

Funding: This research received no external funding.

Institutional Review Board Statement: The study was conducted according to the guidelines of the Declaration of Helsinki, and approved by the Ethics Committee of Kyoto University Graduate School and Faculty of Medicine (R1512, May 2018; R1213, July 2014).

Informed Consent Statement: Informed consent was waived due to the retrospective nature of the study for subjects who participated in previous studies with consent. 
Acknowledgments: Authors thank Kyoko Takakura and Tae Oishifor their contribution on dbPET image acquisition, and Shunsuke Yuge for his help in image preparation.

Conflicts of Interest: Part of data was obtained in a previous study that was supported by Shimadzu Co., Kyoto, Japan, and a Grant-in-Aid for Scientific Research from the Ministry of Education, Culture, Sports, Science and Technology of Japan (C:22591329). The funder had no role in the design of the study; in the collection, analyses, or interpretation of data; in the writing of the manuscript, or in the decision to publish the results.

\section{Appendix A}

Table A1. dbPET lexicon, version 1.0.

\begin{tabular}{|c|c|c|c|}
\hline \multicolumn{4}{|c|}{ Image quality } \\
\hline \multirow{4}{*}{ Noise } & a. & Minimal & Absence of noise or almost no noise \\
\hline & b. & $\begin{array}{l}\text { Mild or limited } \\
\text { to the edge of } \\
\text { FOV }\end{array}$ & Mild noise or noise localized only to the near FOV edge \\
\hline & c. & Moderate & Moderate noise \\
\hline & d. & Marked & Significant noise, which can mask lesions \\
\hline \multirow{4}{*}{ FOV } & a. & Full & $\begin{array}{l}\text { The whole radiotracer-avid fibroglandular gland is completely } \\
\text { contained within the FOV. The entire retromammary space is } \\
\text { identified. }\end{array}$ \\
\hline & b. & Almost full & $\begin{array}{l}\text { Most of the radiotracer-avid fibroglandular gland is included. The } \\
\text { retromammary space is partially interrupted. }\end{array}$ \\
\hline & c. & Partial & $\begin{array}{l}\text { The radiotracer-avid fibroglandular gland is partly out of FOV. The } \\
\text { retromammary space is partly or poorly visualized. }\end{array}$ \\
\hline & d. & Limited & The breast in the FOV is visualized very limitedly. \\
\hline Notes & \multicolumn{3}{|c|}{$\begin{array}{l}\text { If there are special notes (poor positioning, body movement, artifacts, and change of protocols } \\
\text { (radiotracer dose, waiting time, emission scan duration, reconstruction parameters, etc.)), } \\
\text { describe. }\end{array}$} \\
\hline \multicolumn{4}{|c|}{ Background fibroglandular uptake (bFGU) } \\
\hline \multirow{5}{*}{ Extent } & 0. & None & $\begin{array}{l}\text { No radiotracer-avid fibroglandular tissue. When this category is } \\
\text { chosen, subsequent evaluation of intensity and homogeneity of FGU } \\
\text { will be skipped. }\end{array}$ \\
\hline & a. & Small & $\begin{array}{l}\text { A small amount of radiotracer-avid fibroglandular tissue, occupying } \\
\text { approximately }>0 \% \text { to } \leq 25 \% \text { of the assumed fibroglandular tissue in } \\
\text { the breast by volume }\end{array}$ \\
\hline & & $\begin{array}{l}\text { Relatively } \\
\text { small }\end{array}$ & $\begin{array}{l}\text { A relatively small amount of radiotracer-avid fibroglandular tissue, } \\
\text { occupying approximately }>25 \% \text { to } \leq 50 \% \text { of the assumed } \\
\text { fibroglandular tissue in the breast by volume }\end{array}$ \\
\hline & c. & Relatively large & $\begin{array}{l}\text { A relatively large amount of radiotracer-avid fibroglandular tissue, } \\
\text { occupying approximately }>50 \% \text { to } \leq 75 \% \text { of the assumed } \\
\text { fibroglandular tissue in the breast by volume }\end{array}$ \\
\hline & & Large & $\begin{array}{l}\text { A large amount of radiotracer-avid fibroglandular tissue, occupying } \\
\text { approximately }>75 \% \text { to } \leq 100 \% \text { of the assumed fibroglandular tissue } \\
\text { in the breast by volume }\end{array}$ \\
\hline
\end{tabular}


Table A1. Cont.

\begin{tabular}{|c|c|c|c|}
\hline & a. & Faint & Very low intensity (Approximately SUV <1 for ${ }^{18}$ F-FDG). \\
\hline \multirow{3}{*}{ Intensity } & b. & Mild & Low intensity (Approximately SUV $\geq 1$ to $<2$ for ${ }^{18}$ F-FDG) \\
\hline & c. & Moderate & Mildly increased uptake. (Approximately SUV $\geq 2$ to $<3$ for ${ }^{18}$ F-FDG) \\
\hline & d. & Intense & Extremely increased uptake (Approximately SUV $\geq 3$ for ${ }^{18}$ F-FDG) \\
\hline \multirow{3}{*}{ Distribution } & a. & Homogeneous & $\begin{array}{l}\text { Almost uniform and confluent uptake of the radiotracer-avid } \\
\text { fibroglandular tissue }\end{array}$ \\
\hline & b. & Heterogeneous & $\begin{array}{l}\text { Heterogeneous fibroglandular tissue uptake, due to the mixture of fat } \\
\text { (i.e., heterogenous, scattered, island-like or patchy) or uneven uptake } \\
\text { level in the fibroglandular tissue itself. }\end{array}$ \\
\hline & a. & Symmetric & Almost symmetrical pattern between the right and left breasts \\
\hline Symmetry & b. & Asymmetric & Asymmetrical pattern between the right and left breasts \\
\hline Notes & \multicolumn{3}{|c|}{ If there are special notes such as post-mastectomy, post-reconstruction, describe. } \\
\hline \multicolumn{4}{|l|}{ Lesion type } \\
\hline \multicolumn{4}{|c|}{ Focus; Dot-like accumulation. Size threshold is not defined as a criterion, but focus is roughly smaller than $5 \mathrm{~mm}$ as a guide. } \\
\hline \multirow[b]{2}{*}{ Single or multiple } & a. & Single & Single focus \\
\hline & b. & Multiple & Three of more foci, scattered in the breast \\
\hline \multicolumn{4}{|c|}{ Mass uptake (MU); Three-dimensionally mass-like uptake } \\
\hline \multirow{4}{*}{ Shape } & a. & Oval & $\begin{array}{l}\text { Elliptical or egg-shaped uptake (may have up to } 2 \text { mild undulations } \\
\text { with a dull angle) }\end{array}$ \\
\hline & & Round & $\begin{array}{l}\text { Spherical, circular, round uptake (may have up to } 2 \text { mild undulations } \\
\text { with a dull angle) }\end{array}$ \\
\hline & c. & Lobulated & $\begin{array}{l}\text { Lobulated uptake with clear undulations with sharp angles, or } 3 \text { or } \\
\text { more undulations }\end{array}$ \\
\hline & d. & Irregular & $\begin{array}{l}\text { Uptake with irregular margins (sawtooth, spiculated, angular, or } \\
\text { indistinct) or lesions that develop irregular extension }\end{array}$ \\
\hline \multirow{3}{*}{ Internal pattern } & a. & Homogeneous & Almost homogeneous accumulation \\
\hline & & Heterogeneous & Heterogeneous accumulation \\
\hline & c. & Rim uptake & $\begin{array}{l}\text { Rim-shaped accumulation with a loss or marked reduction of } \\
\text { accumulation in the central part. The rim includes full rim and partial } \\
\text { rim. }\end{array}$ \\
\hline
\end{tabular}


Table A1. Cont.

\begin{tabular}{|c|c|c|c|}
\hline $\begin{array}{l}\text { Non-mass uptak } \\
\text { focus }\end{array}$ & & the backgroun & ibroglandular tissue, and has a shape that cannot be called a mass or \\
\hline & a. & Focal & Less than $25 \%$ of quadrant or less than $2 \mathrm{~cm}$ in diameter \\
\hline & b. & Linear & Linear toward the nipple. It may be tubular or branched. \\
\hline & c. & Segmental & $\begin{array}{l}\text { Distribution of triangles with one apex towards the nipple side, } \\
\text { suggesting distribution of glandular lobes and ducts }\end{array}$ \\
\hline Distribution & d. & Regional & $25 \%$ or more of quadrant or $2 \mathrm{~cm}$ or more in diameter \\
\hline & e. & $\begin{array}{l}\text { Multiple } \\
\text { regions }\end{array}$ & $\begin{array}{l}\text { Multiple uptake areas in } 3 \text { or more locations, independent from the } \\
\text { distribution of glandular lobes. Focus can be included, but uptake } \\
\text { pattern at least one location should be NMU. }\end{array}$ \\
\hline & f. & Diffuse & Diffuse accumulation \\
\hline & a. & Homogeneous & Almost homogeneous \\
\hline Internal pattern & b. & Heterogeneous & Heterogeneous \\
\hline
\end{tabular}

Common assessment for lesion

\begin{tabular}{|c|c|c|c|}
\hline \multirow{12}{*}{ Location } & \multirow{2}{*}{ Laterality } & \multicolumn{2}{|l|}{ Right } \\
\hline & & \multicolumn{2}{|l|}{ Left } \\
\hline & \multirow{5}{*}{ Quadrant } & UOQ & Upper outer quadrant \\
\hline & & LOQ & Lower outer quadrant \\
\hline & & UIQ & Upper inner quadrant \\
\hline & & LIQ & Lower inner quadrant \\
\hline & & Retroareolar & Around or posterior to the nipple \\
\hline & Clock face & & Clock-face position \\
\hline & \multirow{3}{*}{ Depth } & Anterior & $1 / 3$ anterior of the breast within FOV \\
\hline & & Middle & $1 / 3$ middle of the breast within FOV \\
\hline & & Posterior & $1 / 3$ posterior of the breast within FOV \\
\hline & Distance from the nipple & & Distance of the lesion from the nipple \\
\hline \multirow{4}{*}{\multicolumn{2}{|c|}{ Qualitative uptake intensity }} & a. None & No abnormal accumulation \\
\hline & & b. Mild & Low intensity (Approximately SUV < 2 for ${ }^{18}$ F-FDG). \\
\hline & & c. Moderate & Moderate uptake (Approximately SUV $\geq 2,<3$ for ${ }^{18}$ F-FDG) \\
\hline & & d. Intense & Increased uptake (Approximately SUV $\geq 3$ for ${ }^{18} \mathrm{~F}$-FDG) \\
\hline
\end{tabular}


Table A1. Cont.

\begin{tabular}{|c|c|c|}
\hline Quantitative uptake intensity & & Qualitative radiotracer uptake values, i.e., SUV \\
\hline \multirow{5}{*}{ Associated findings } & Nipple retraction & \\
\hline & Skin retraction & \\
\hline & Nipple uptake & \\
\hline & Skin uptake & \\
\hline & $\begin{array}{l}\text { Pectoral } \\
\text { muscle/chest wall } \\
\text { uptake }\end{array}$ & \\
\hline \multicolumn{3}{|l|}{ Special findings } \\
\hline & Cyst-like uptake & $\begin{array}{l}\text { A clear circular uptake defect with or without surrounding uptake. } \\
\text { This category is selected when cyst or fat necrosis is strongly } \\
\text { suspected or known. }\end{array}$ \\
\hline & Breast implant & Accumulation defect due to implant \\
\hline
\end{tabular}

dbPET, dedicated breast positron emission tomography; FOV, field of view; SUV, standard uptake value; ${ }^{18} \mathrm{~F}-\mathrm{FDG},{ }^{18} \mathrm{~F}$-fluorodeoxyglucose.

\section{References}

1. Satoh, Y.; Kawamoto, M.; Kubota, K.; Murakami, K.; Hosono, M.; Senda, M.; Sasaki, M.; Momose, T.; Ito, K.; Okamura, T.; et al. Clinical practice guidelines for high-resolution breast PET, 2019 Edition. Ann. Nucl. Med. 2021, 35, 406-414. [CrossRef]

2. Miyake, K.K.; Nakamoto, Y.; Togashi, K. Current Status of Dedicated Breast PET Imaging. Curr. Radiol. Rep. 2016, 4, 16. [CrossRef]

3. Thompson, C.J.; Murthy, K.; Picard, Y.; Weinberg, I.N.; Mako, R. Positron emission mammography (PEM): A promising technique for detecting breast cancer. IEEE Trans. Nucl. Sci. 1995, 42, 1012-1017. [CrossRef]

4. Miyake, K.K.; Matsumoto, K.; Inoue, M.; Nakamoto, Y.; Kanao, S.; Oishi, T.; Kawase, S.; Kitamura, K.; Yamakawa, Y.; Akazawa, A.; et al. Performance Evaluation of a New Dedicated Breast PET Scanner Using NEMA NU4-2008 Standards. J. Nucl. Med. 2014, 55, 1198-1203. [CrossRef] [PubMed]

5. Moliner, L.; González, A.J.; Soriano, A.; Sánchez, F.; Correcher, C.; Orero, A.; Carles, M.; Vidal, L.F.; Barberá, J.; Caballero, L.; et al. Design and evaluation of the MAMMI dedicated breast PET. Med. Phys. 2012, 39, 5393-5404. [CrossRef]

6. $\quad$ Eo, J.S.; Chun, I.K.; Paeng, J.C.; Kang, K.W.; Lee, S.M.; Han, W.; Noh, D.Y.; Chung, J.K.; Lee, D.S. Imaging sensitivity of dedicated positron emission mammography in relation to tumor size. Breast 2012, 21, 66-71. [CrossRef] [PubMed]

7. Kalinyak, J.E.; Berg, W.A.; Schilling, K.; Madsen, K.S.; Narayanan, D.; Tartar, M. Breast cancer detection using high-resolution breast PET compared to whole-body PET or PET/CT. Eur. J. Nucl. Med. Mol. Imaging 2014, 41, 260-275. [CrossRef] [PubMed]

8. Yamamoto, Y.; Ozawa, Y.; Kubouchi, K.; Nakamura, S.; Nakajima, Y.; Inoue, T. Comparative analysis of imaging sensitivity of positron emission mammography and whole-body PET in relation to tumor size. Clin. Nucl. Med. 2015, 40, 21-25. [CrossRef]

9. Yano, F.; Itoh, M.; Hirakawa, H.; Yamamoto, S.; Yoshikawa, A.; Hatazawa, J. Diagnostic Accuracy of Positron Emission Mammography with 18F-Fluorodeoxyglucose in Breast Cancer Tumor of Less than $20 \mathrm{~mm}$ in Size. Asia Ocean J. Nucl. Med. Biol. 2019, 7, 13-21. [CrossRef] [PubMed]

10. Sueoka, S.; Sasada, S.; Masumoto, N.; Emi, A.; Kadoya, T.; Okada, M. Performance of dedicated breast positron emission tomography in the detection of small and low-grade breast cancer. Breast Cancer Res. Treat. 2021, 187, 125-133. [CrossRef]

11. D'Orsi, C.; Sickles, E.; Mendelson, E.; Morris, E. Breast Imaging Reporting and Data System: ACR BI-RADS—Breast Imaging Atlas, 5th ed.; American College of Radiology: Reston, VA, USA, 2013.

12. Narayanan, D.; Madsen, K.S.; Kalinyak, J.E.; Berg, W.A. Interpretation of positron emission mammography: Feature analysis and rates of malignancy. AJR Am. J. Roentgenol. 2011, 196, 956-970. [CrossRef] [PubMed]

13. Satoh, Y.; Motosugi, U.; Omiya, Y.; Onishi, H. Unexpected Abnormal Uptake in the Breasts at Dedicated Breast PET: Incidentally Detected Small Cancers or Nonmalignant Features? AJR Am. J. Roentgenol. 2019, 212, 443-449. [CrossRef] [PubMed]

14. Sasada, S.; Masumoto, N.; Kimura, Y.; Emi, A.; Kadoya, T.; Okada, M. Classification of Abnormal Findings on Ring-type Dedicated Breast PET for the Detection of Breast Cancer. Anticancer Res. 2020, 40, 3491-3497. [CrossRef] [PubMed]

15. Masumoto, N.; Kadoya, T.; Sasada, S.; Emi, A.; Arihiro, K.; Okada, M. Intratumoral heterogeneity on dedicated breast positron emission tomography predicts malignancy grade of breast cancer. Breast Cancer Res. Treat. 2018, 171, 315-323. [CrossRef]

16. Sakaguchi, R.; Kataoka, M.; Kanao, S.; Miyake, K.K.; Nakamoto, Y.; Sugie, T.; Toi, M.; Mikami, Y.; Togashi, K. Distribution pattern of FDG uptake using ring-type dedicated breast PET in comparison to whole-body PET/CT scanning in invasive breast cancer. Ann. Nucl. Med. 2019, 33, 570-578. [CrossRef] [PubMed]

17. Delbeke, D.; Coleman, R.E.; Guiberteau, M.J.; Brown, M.L.; Royal, H.D.; Siegel, B.A.; Townsend, D.W.; Berland, L.L.; Parker, J.A.; Hubner, K.; et al. Procedure guideline for tumor imaging with 18 F-FDG PET/CT 1.0. J. Nucl. Med. 2006, 47, 885-895. [PubMed]

18. Boellaard, R.; Delgado-Bolton, R.; Oyen, W.J.G.; Giammarile, F.; Tatsch, K.; Eschner, W.; Verzijlbergen, F.J.; Barrington, S.F.; Pike, L.C.; Weber, W.A.; et al. FDG PET/CT: EANM procedure guidelines for tumour imaging: Version 2.0. Eur. J. Nucl. Med. Mol. Imaging 2015, 42, 328-354. [CrossRef] [PubMed] 
19. Berg, W.A.; Madsen, K.S.; Schilling, K.; Tartar, M.; Pisano, E.D.; Larsen, L.H.; Narayanan, D.; Ozonoff, A.; Miller, J.P.; Kalinyak, J.E. Breast cancer: Comparative effectiveness of positron emission mammography and MR imaging in presurgical planning for the ipsilateral breast. Radiology 2011, 258, 59-72. [CrossRef] [PubMed]

20. Nishimatsu, K.; Nakamoto, Y.; Miyake, K.K.; Ishimori, T.; Kanao, S.; Toi, M.; Togashi, K. Higher breast cancer conspicuity on dbPET compared to WB-PET/CT. Eur. J. Radiol. 2017, 90, 138-145. [CrossRef] [PubMed]

21. Conners, A.L.; Hruska, C.B.; Tortorelli, C.L.; Maxwell, R.W.; Rhodes, D.J.; Boughey, J.C.; Berg, W.A. Lexicon for standardized interpretation of gamma camera molecular breast imaging: Observer agreement and diagnostic accuracy. Eur. J. Nucl. Med. Mol. Imaging 2012, 39, 971-982. [CrossRef] [PubMed] 